1985,14030

Center for Turbulence Research

Proceedings of the Summer Program 1994

\title{
The decay of axisymmetric homogeneous turbulence
}

\author{
By J. R. Chasnov ${ }^{1}$
}

The decay of a homogeneous turbulence generated by an axisymmetric distribution of random impulsive forces acting at the initial instant is considered. The impulsive forces may be either parallel or perpendicular to the symmetry axis. For impulsive forces which result in a $k^{4}$ low wavenumber energy spectrum of the turbulence, it is determined that the flow approaches isotropy on all scales of motion at longtimes provided the Reynolds number is large. However, for the type of impulsive forces originally proposed by Saffman [J. Fluid Mech 27, 581 (1967); Phys. Fluids $10,1349(1967)]$ in which a $k^{2}$ low wavenumber energy spectrum is produced, the turbulence approaches isotropy only at the smallest scales and remains significantly anisotropic at the largest and energy-containing scales. Nevertheless, a similarity state of the flow field establishes itself asymptotically, in which the kinetic energy per unit mass of the turbulence decays as $t^{-6 / 5}$.

\section{Introduction}

An asymptotic similarity state of decaying isotropic turbulence was predicted by Kolmogorov (1941) based on a supposed dynamical invariant of the flow field (Loitsianski, 1939). It was later shown by theoretical arguments (Batchelor and Proudman, 1956) that the Loitsianski integral is in fact not invariant, and under certain conditions of turbulence generation may even diverge (Saffman, 1967a). For this Saffman-type flow, a new invariant was discovered and a similarity state of decaying homogeneous turbulence at high Reynolds numbers was postulated based on this invariant (Saffman, 1967b). Recent large-eddy simulations of isotropic turbulence (Chasnov, 1994) have confirmed the existence of this exact similarity state to within a few percent. Here, we extend these recent large-eddy simulations of isotropic turbulence to a decaying statistically axisymmetric flow field. Our main objective here is to determine if a long-time, high Reynolds number similarity state of decaying axisymmetric turbulence occurs. Of course, if the axisymmetric turbulence approaches isotropy asymptotically, then the earlier found similarity state would necessarily be recovered.

However, a complete return-to-isotropy of the turbulence is impossible for the Saffman-type of flow since the large-scale structure of the turbulence is preserved for all times. For instance, if the initial flow field was anisotropic due to some anisotropy in the method of turbulence generation, then this initial anisotropy would remain in the large-scales of the flow for all times. Of course, the small scales of the flow

1 The Hong Kong University of Science and Technology
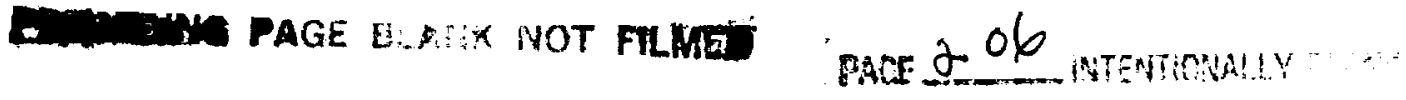
may become isotropic, but the main scales of interest to us here with regards to an asymptotic similarity state are those which contain most of the energy of the turbulence; these scales may be more directly affected by the presence of large-scale anisotropy.

Previous closure calculations and numerical simulations have studied the decay of an initially axisymmetric turbulence (Herring, 1974; Schumann and Herring, 1976; Schumann and Patterson, 1978) in the context of the return-to-isotropy problem. The direct numerical simulations performed in the latter two works were necessarily limited to low Reynolds numbers, and the computer resources available at those times allowed only a resolution of $32^{3}$. Nevertheless, an approach of the anisotropic initial state towards isotropy was indicated.

This earlier work postulated arbitrary initial states of the axisymmetric turbulence without considering whether or not the large-scale structure of the flow was invariant. Here, following closely the work of Saffman (1967a), we assume that our initial flow fields are generated by random axisymmetric impulsive forces at the initial instant such as may be generated by laminar flow passing through a vigorously shaken grid. We consider impulsive forces which are either parallel or perpendicular to the symmetry axis. Both the Saffman-type flow and the original Batchelor-Proudman flow will also be considered. In the latter type of flow, the large-scale structure of the turbulence is not permanent due to nonlinear transfer from small-to-large scales, so that a return-to-isotropy of all scales of motion is possible.

\section{Generation by random axisymmetric impulsive forces}

We consider an infinite incompressible fluid initially at rest to which is applied random impulsive forces at the initial instant. The continuity and Navier-Stokes equations which govern the fluid motion are

$$
\begin{gathered}
\nabla \cdot \mathbf{u}=0 \\
\frac{\partial \mathbf{u}}{\partial t}+\mathbf{u} \cdot \nabla \mathbf{u}=-\frac{\nabla p}{\rho_{0}}+\nu \nabla^{2} \mathbf{u}+\mathbf{f} \delta(t)
\end{gathered}
$$

where $\mathbf{f}$ is the impulsive force field per unit mass distributed throughout the fluid; we assume that this vector force field is a stationary random function of the coordinate $\mathbf{x}$. Its multiplier $\delta(t)$ is the usual Dirac-delta function, which confines the impulse to the initial instant. Eq. (2) may be integrated to $t=0^{+}$at which time the impulse ceases. Denoting the Fourier transform of $u_{i}(\mathbf{x}, t)$ by $\hat{u}_{i}(\mathbf{k}, t)$ and the Fourier transform of $f_{i}(\mathbf{x})$ by $\hat{f}_{i}(\mathbf{k})$, the Fourier components of the resulting impulse-generated velocity field at $t=0^{+}$is given by

$$
\hat{u}_{i}\left(\mathbf{k}, 0^{+}\right)=P_{i j}(\mathbf{k}) \hat{f}_{j}(\mathbf{k}),
$$

where $P_{i j}(\mathbf{k})=\delta_{i j}-k_{i} k_{j} ; / k^{2}$, and $\delta_{i j}$ is the usual Kronecker-delta. The projection operator $P_{i j}(\mathbf{k})$ appearing on the right hand side of (3) occurs because the pressure forces respond to the initial impulse so as to maintain the incompressibility of the 
fluid. The spectral tensor $\Phi_{i j}(\mathbf{k}, t)$ of the velocity correlation after the fluid is acted on by the impulsive forces is thus given by

$$
\Phi_{i j}\left(\mathbf{k}, 0^{+}\right)=P_{i m}(\mathbf{k}) P_{j n}(\mathbf{k}) \mathcal{M}_{m n}(\mathbf{k})
$$

where $\mathcal{M}_{m n}(\mathbf{k})$ is the spectral tensor of the force correlation, which is assumed to exist. The result (4) was previously obtained by Saffman (1967a). We now specialize to two particular impulsive force distributions which exhibit statistical axisymmetry and could conceivably be generated in a laboratory experiment.

\subsection{Impulse parallel to the symmetry axis}

The physical situation we envision is that of grid-generated turbulence in which the grid is randomly shaken along the direction of the mean fluid velocity. The impulsive force of the grid on the fluid should ideally be a stationary random function of time and of the coordinates in the plane perpendicular to the mean velocity. Taylor's hypothesis can then be invoked to relate the grid turbulence experiment to the decaying homogeneous turbulence considered here.

We shall, without loss of generality, assume symmetry about the $x_{3}$ axis. The spectral tensor of the force correlation corresponding to the above physical experiment can be written as

$$
\mathcal{M}_{i j}(\mathbf{k})=\delta_{i 3} \delta_{j 3} M(\mathbf{k}),
$$

and the corresponding spectral tensor of the velocity correlation generated at the initial instant thus becomes

$$
\Phi_{i j}\left(\mathbf{k}, 0^{+}\right)=P_{i 3}(\mathbf{k}) P_{j 3}(\mathbf{k}) M(\mathbf{k}) .
$$

It will be convenient to write the spectral tensor in terms of two unit vectors which are perpendicular to $\mathbf{k}$ and thus explicitly satisfy the continuity equation (Herring, 1974). These unit vectors are defined as

$$
\mathbf{e}^{(1)}(\mathbf{k})=\frac{\mathbf{k} \times \mathbf{n}}{|\mathbf{k} \times \mathbf{n}|}, \quad \mathbf{e}^{(2)}(\mathbf{k})=\frac{\mathbf{k} \times \mathbf{e}^{(1)}(\mathbf{k})}{\left|\mathbf{k} \times \mathbf{e}^{(1)}(\mathbf{k})\right|}
$$

where $\mathbf{n}=(0,0,1)$ is the unit vector along the symmetry axis. Using the relation $P_{i j}(\mathbf{k})=e_{i}^{(1)}(\mathbf{k}) e_{j}^{(1)}(\mathbf{k})+e_{i}^{(2)}(\mathbf{k}) e_{j}^{(2)}(\mathbf{k})$, it is easy to show that $(6)$ becomes

$$
\Phi_{i j}\left(\mathbf{k}, 0^{+}\right)=\left(1-\frac{k_{3}^{2}}{k^{2}}\right) e_{i}^{(2)}(\mathbf{k}) e_{j}^{(2)}(\mathbf{k}) M(\mathbf{k}) .
$$

\subsection{Impulse perpendicular to the symmetry axis}

Here, the grid-generated turbulence experiment we envision is the same as above except that now the grid is randomly shaken in the direction perpendicular to the mean fluid velocity. To maintain the statistical axisymmetry of the force distribution and the resulting flow field, we assume that the random impulsive forces acting on 
the fluid have no preferred direction in the plane perpendicular to the symmetry axis. The spectral tensor of the force correlation may now be written as

$$
\mathcal{M}_{i j}(\mathbf{k})=\frac{1}{2}\left(\delta_{i j}-\delta_{i 3} \delta_{j 3}\right) M(\mathbf{k}),
$$

where the factor of $1 / 2$ has been inserted for later convenience. The spectral tensor of the velocity correlation at the initial instant thus becomes

$$
\Phi_{i j}\left(\mathbf{k}, 0^{+}\right)=\frac{1}{2}\left[P_{i j}(\mathbf{k})-P_{i 3}(\mathbf{k}) P_{j 3}(\mathbf{k})\right] M(\mathbf{k})
$$

which in terms of the $e$-basis can be written as

$$
\Phi_{i j}\left(\mathbf{k}, 0^{+}\right)=\frac{1}{2}\left[e_{i}^{(1)}(\mathbf{k}) e_{j}^{(1)}(\mathbf{k})+\frac{k_{3}^{2}}{k^{2}} e_{i}^{(2)}(\mathbf{k}) e_{j}^{(2)}(\mathbf{k})\right] M(\mathbf{k})
$$

\subsection{Specification of the spectral tensor of the force correlation}

The initial spectrum of the homogeneous turbulence flow field immediately after generation by the random impulsive forces is now completely specified provided the form of the spectrum $M(\mathbf{k})$ of the force correlation is known. For simplicity, we assume that $M$ is a function only of the wavenumber magnitude $k$. The general form we choose for $M(k)$ is

$$
M(k)=\frac{3}{8 \pi} a_{s} u_{0}^{2} k_{p}^{-3}\left(\frac{k}{k_{p}}\right)^{s-2} \exp \left[-\frac{1}{2} s\left(\frac{k}{k_{p}}\right)^{2}\right],
$$

where $u_{0}$ is the root-mean-square velocity of the fluid immediately after generation by both types of impulsive force distributions, $k_{p}$ is the wavenumber at which the initial spherically integrated energy spectrum of the turbulence is maximum, and $s=2$ or 4 , corresponding either to a Saffman-type flow (Saffman, 1967) or a Batchelor-Proudman-type flow (Batchelor \& Proudman, 1956), further details of which will be presented in the next Section. The normalization constant $a_{s}$ is given by

$$
a_{s}=\sqrt{\frac{2}{\pi}} \frac{s^{\frac{1}{2}(s+1)}}{1 \cdot 3 \cdots \cdots(s-1)} .
$$

\section{Possible similarity states}

We consider whether a similarity state of the decaying turbulence develops asymptotically in time provided the Reynolds number of the flow is large. Saffman (1967a) has shown that when $s=2$ in (12), the form of the spectral tensor, (8) or (11), persists for all times near $k=0$. It is customary to define the associated dynamical invariant of the flow field in terms of the leading-order spectral coefficient of the spherically-integrated energy spectrum $E(k)$. By spherically-integrating either (8) or (11), using (12), the energy spectrum at the initial instant can be shown to have the form 


$$
E\left(k, 0^{+}\right)=\frac{1}{2} a_{s} u_{0}^{2} k_{p}^{-1}\left(\frac{k}{k_{p}}\right)^{s} \exp \left[-\frac{1}{2} s\left(\frac{k}{k_{p}}\right)^{2}\right],
$$

so that when $s=2, E(k, t)$ behaves for all times near $k=0$ as $E(k, t) \sim 2 \pi k^{2} B_{0}$, where

$$
B_{0}=\frac{a_{2} u_{0}^{2}}{4 \pi k_{p}^{3}}
$$

is a dynamical invariant. Normalization constants in (12) have been chosen so that (14) agrees with our previous large-eddy simulations of decaying isotropic turbulence (Chasnov, 1994).

A similarity state of the turbulence which is now based on the invariant $B_{0}$ leads directly to the decay of the mean-square kinetic energy per unit mass as (Saffman, 1967b)

$$
\left\langle\mathbf{u}^{2}\right\rangle \propto B_{0}^{\frac{2}{6}} t^{-\frac{6}{8}}
$$

The power-law exponent $-6 / 5$ was verified to within a few percent in a decaying isotropic turbulence (Chasnov, 1994). Saffman (1967a) has further speculated that the decay law given by (16) may be valid for a decaying turbulence which does not exhibit isotropic symmetry, and one of the purposes of the present work is to test this hypothesis.

When the initial impulsive force distribution is such that $s=4$ in (14), the spherically integrated three-dimensional energy spectrum of the turbulence follows $E(k) \sim 2 \pi k^{4} B_{2}$ near $k=0$, where now $B_{2}=B_{2}(t)$ is a function of time, as shown analytically by Batchelor and Proudman (1957). Lesieur and collaborators (Lesieur, 1990), following earlier work of Kolmogorov (1941) and Comte-Bellot and Corrsin (1966), have postulated a similarity state of the flow field based on $B_{2}(t)$, which was then confirmed within the framework of two-point closure calculations. A numerical calculation of $B_{2}(t)$ was also performed by computing an ensemble average of a large number (1024) of large-eddy simulations of decaying isotropic turbulence (Chasnov, 1993). From the two-point closure calculations and largeeddy simulations, the time-dependence of $B_{2}(t)$ has been shown to be weak relative to the overall turbulence decay (the large-eddy simulations of isotropic turbulence determined approximately $\left.B_{2}(t) \sim t^{0.25}\right)$. The decay of the mean-square kinetic energy per unit mass may thus be written as

$$
\left\langle\mathbf{u}^{2}\right\rangle \propto B_{2}^{\frac{3}{3}} t^{-\frac{10}{7}}
$$

where the power-law exponent $-10 / 7$ is only approximate (a more precise exponent of -1.36 results when the time-dependence of $B_{2}$ is taken into account).

Of special interest to the present work is the approach of the initially axisymmetric turbulence to isotropy. As a simple measure of the anisotropy of the energy containing scales, we define the parameter $\gamma$ to be 


$$
\gamma(t)=\frac{2\left\langle u_{3}^{2}\right\rangle}{\left\langle u_{1}^{2}+u_{2}^{2}\right\rangle} .
$$

The value of $\gamma$ is easily computed for the flow fields generated at the initial instant: we determine that $\gamma(0)=8$ or $\gamma(0)=2 / 9$ for impulsive forces which are parallel or perpendicular to the symmetry axis, respectively. Non-linear transfer processes may reasonably be expected to bring the turbulence closer to statistical isotropy $(\gamma=1)$ at later times.

We have already noted an interesting difference between flows with $s=2$ or 4 in (12) which may affect the eventual return-to-isotropy of the flow fields. When $s=2$ in (12), the permanence of the large-scale structure of the flow precludes the entire flow from ever becoming isotropic. However, the parameter $\gamma$ is primarily a measure of the anisotropy of the energy-containing scales so it is yet unclear whether these scales will approach isotropy, or whether the anisotropy present in the largest scales will affect the asymptotic state of the energy-containing scales. When $s=4$ in (12), the large-scale structure of the turbulence is no longer preserved for all times since the low wavenumber coefficients of the spectral tensor are no longer invariant. It is plausible, although perhaps not entirely obvious, that non-linear interactions can in this case produce an isotropic flow field asymptotically in time for sufficiently large Reynolds numbers.

Large-eddy simulations presented in the next Section will shed further light on the existence of an asymptotic similarity state in decaying axisymmetric turbulence, and the approach (or non-approach) to isotropy of the flow fields.

\section{Large-eddy simulation}

The large-eddy simulations presented here were performed using a pseudospectral code for turbulence in a periodic box of length $2 \pi$ (Rogallo, 1981), and a spectral eddy-viscosity subgrid-scale model (Kraichnan, 1976; Chollet and Lesieur, 1981). More details about the large-eddy simulation technique can be found in Chasnov (1994). Four $128^{3}$ resolution simulations were performed corresponding to an impulse parallel or perpendicular to the symmetry axis, and $s=2$ or 4 in (12) and (14). We also choose $u_{0}=1$ and $k_{p}=50$. The relatively large value of $k_{p}$ allows a similarity state to develop before the integral scales of the flow grow to a size comparable to the periodicity length.

Particular realizations of the impulsive-force-generated velocity fields are constructed as follows. By virtue of the continuity equation, the Fourier components of the velocity field may be projected onto the two unit vectors perpendicular to $k$ given by ( 7$)$ :

$$
u_{i}(\mathbf{k})=\phi_{1}(\mathbf{k}) e_{i}^{(1)}(\mathbf{k})+\phi_{2}(\mathbf{k}) e_{i}^{(2)}(\mathbf{k}) .
$$

When the impulsive forces are parallel to the symmetry axis, the spectral tensor at $t=0^{+}$is given by (8) with $M(k)$ given by (12), and we construct random fields which satisfy $(8)$ by setting 


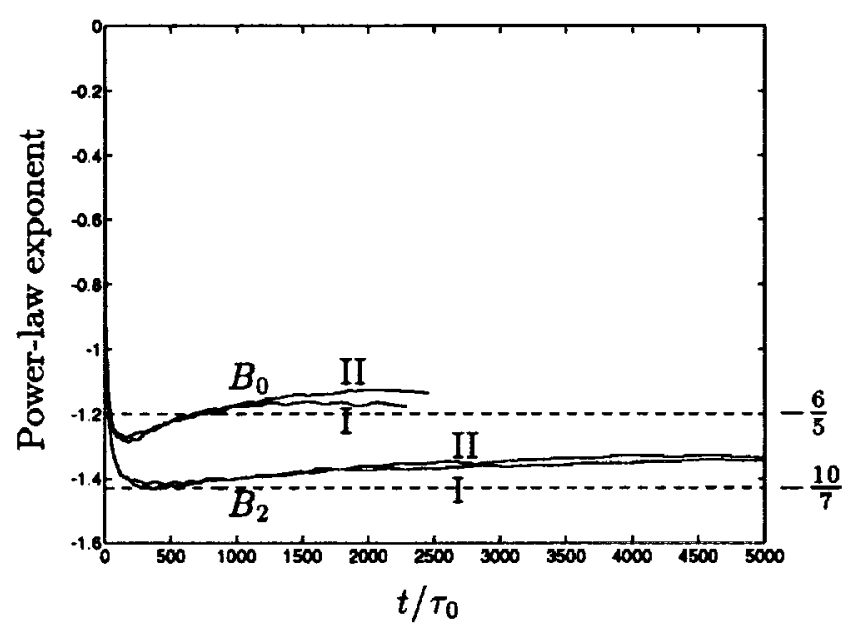

Figure 1. Time-evolution of the power-law exponent of $\left\langle\mathbf{u}^{2}\right\rangle$. The solid lines correspond to the results of the four large-eddy simulations and the dashed lines correspond to the Saffman and Kolmogorov laws discussed in Section 3. The curves are labelled by $B_{0}$ and $B_{2}$ corresponding to $s=2$ or 4 in (12), respectively; and by I and II corresponding to impulsive forces parallel or perpendicular to the symmetry axis, respectively.

$$
\phi_{1}(\mathbf{k})=0, \quad \phi_{2}(\mathbf{k})=\left[\left(1-\frac{k_{3}^{2}}{k^{2}}\right) M(k)\right]^{\frac{1}{2}} \exp i 2 \pi \theta,
$$

where $\theta$ is a uniformly distributed random number between 0 and 1 , chosen independently for each $\mathbf{k}$ subject to the complex conjugate symmetry of the Fourier components of the velocity field. Similarly, when the impulsive forces are perpendicular to the symmetry axis, the relevant equations are (11) and (12), and the random fields are chosen as

$$
\phi_{1}(\mathbf{k})=\left[\frac{M(k)}{2}\right]^{\frac{1}{2}} \exp i 2 \pi \theta_{1}, \quad \phi_{2}(\mathbf{k})=\frac{k_{3}}{k}\left[\frac{M(k)}{2}\right]^{\frac{1}{2}} \exp i 2 \pi \theta_{2},
$$

with $\theta_{1}$ and $\theta_{2}$ random numbers as above. The three components of the velocity field may then be determined directly from (20) and (21) using (19). The particular realization of the velocity field constructed above is a generalization of the method proposed by Rogallo (1981) for isotropic turbulence.

In Fig. 1, we present the time-evolution of the power-law exponent (logarithmic derivative) of $\left\langle\mathbf{u}^{2}\right\rangle$. Time is normalized in terms of the initial large-eddy turnover time $\tau_{0}$, where $\tau_{0}=L_{0} / u_{0}$, and $L_{0}$ is the initial spherically-averaged integral scale of the flow, given by $L_{0}=\sqrt{\pi} / k_{p}$ for $s=2$ and $L_{0}=2 \sqrt{2 \pi} / 3 k_{p}$ for $s=4$. The curves are labeled by $B_{0}$ or $B_{2}$, the leading-order spectral coefficients of the energy spectrum corresponding to $s=2$ or 4 in (12), and the Roman number I or 

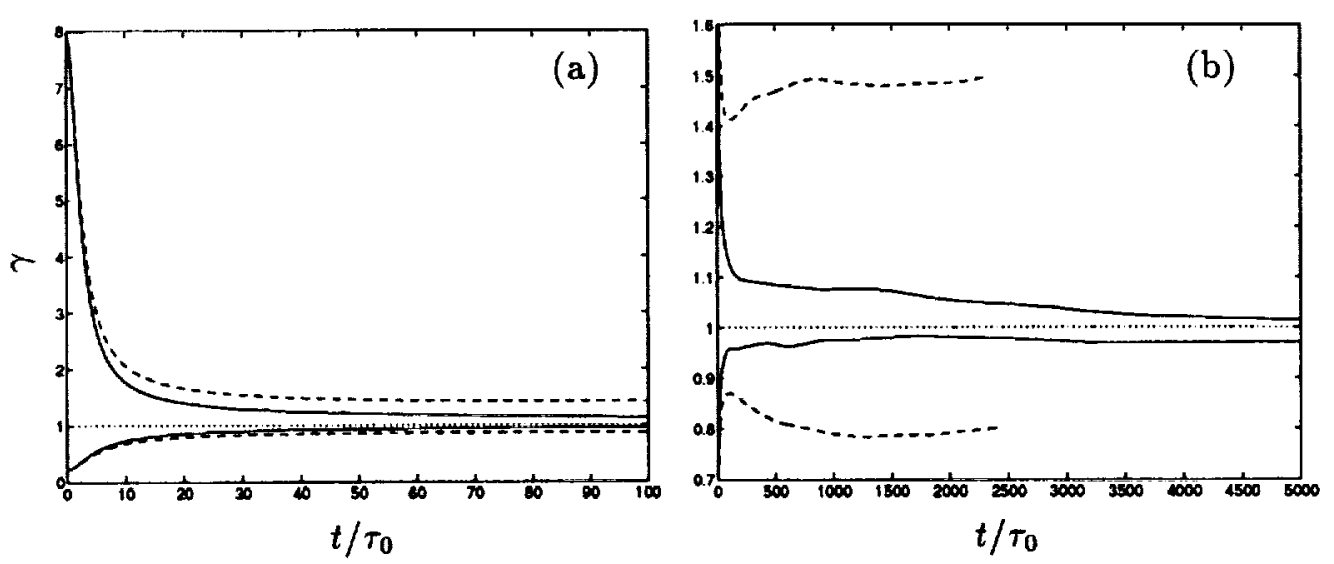

FIgURE 2. Time-evolution of the anisotropy factor $\gamma$, defined in (18). The dashed and solid lines correspond to $s=2$ and $s=4$ in (12), respectively; $\gamma=1$ for an isotropic turbulence. Curves above or below $\gamma=1$ correspond to impulsive forces parallel or perpendicular to the symmetry axis, respectively. (a) Short time evolution to $t / \tau_{0}=100$; (b) Long time evolution to $t / \tau_{0}=5000$.

II, corresponding to whether the impulsive forces are parallel or perpendicular to the symmetry axis, respectively. The dashed lines are the exact and approximate results discussed in Section 3. It is immediately apparent that similarity states in good agreement with either the Saffman and Kolmogorov laws develop in all of the simulations.

An evolution of the anisotropy parameter $\gamma(t)$ as defined in Eq. (18), for short- and long-time evolutions, is shown in Fig. 2. Over short-time evolutions, it is apparent that all four of the flow fields rapidly become more isotropic than the initial state. However, consideration of the long-time asymptotics of $\gamma$ clearly demonstrates that only when $s=4$ (solid-lines) does $\gamma$ approach its isotropic value of unity, whereas when $s=2$ (dashed- lines), $\gamma$ deviates substantially from unity at the latest times. In fact, the asymptotic values of $\gamma$ for these Saffman-type flows are approximately $\gamma \rightarrow 1.5$ or $\gamma \rightarrow 0.8$ for impulsive forces parallel or perpendicular to the symmetry axis, respectively.

The anisotropy of turbulent eddies should depend on their length scales. To observe this effect, we apply the projection of the velocity field defined in (19) to construct the following spherically-integrated spectral functions:

$$
F_{1}(k, t)=4 \pi k^{2}\left\langle\phi_{1}(\mathbf{k}, t) \phi_{1}^{*}(\mathbf{k}, t)\right\rangle, \quad F_{2}(k, t)=4 \pi k^{2}\left\langle\phi_{2}(\mathbf{k}, t) \phi_{2}^{*}(\mathbf{k}, t)\right\rangle
$$

where $*$ denotes the complex conjugate, and the angular brackets used here denote an average over a spherical shell (of unit thickness) in wave space. The usual energy spectrum is obtained from $E(k, t)=\frac{1}{2}\left(F_{1}(k, t)+F_{2}(k, t)\right)$. In an isotropic turbulence $F_{1}(k, t)=F_{2}(k, t)$, and this will serve as an indication of isotropy for scales characterized by a wavenumber magnitude $k$. In Figs. 3(a) and 3(b) we 

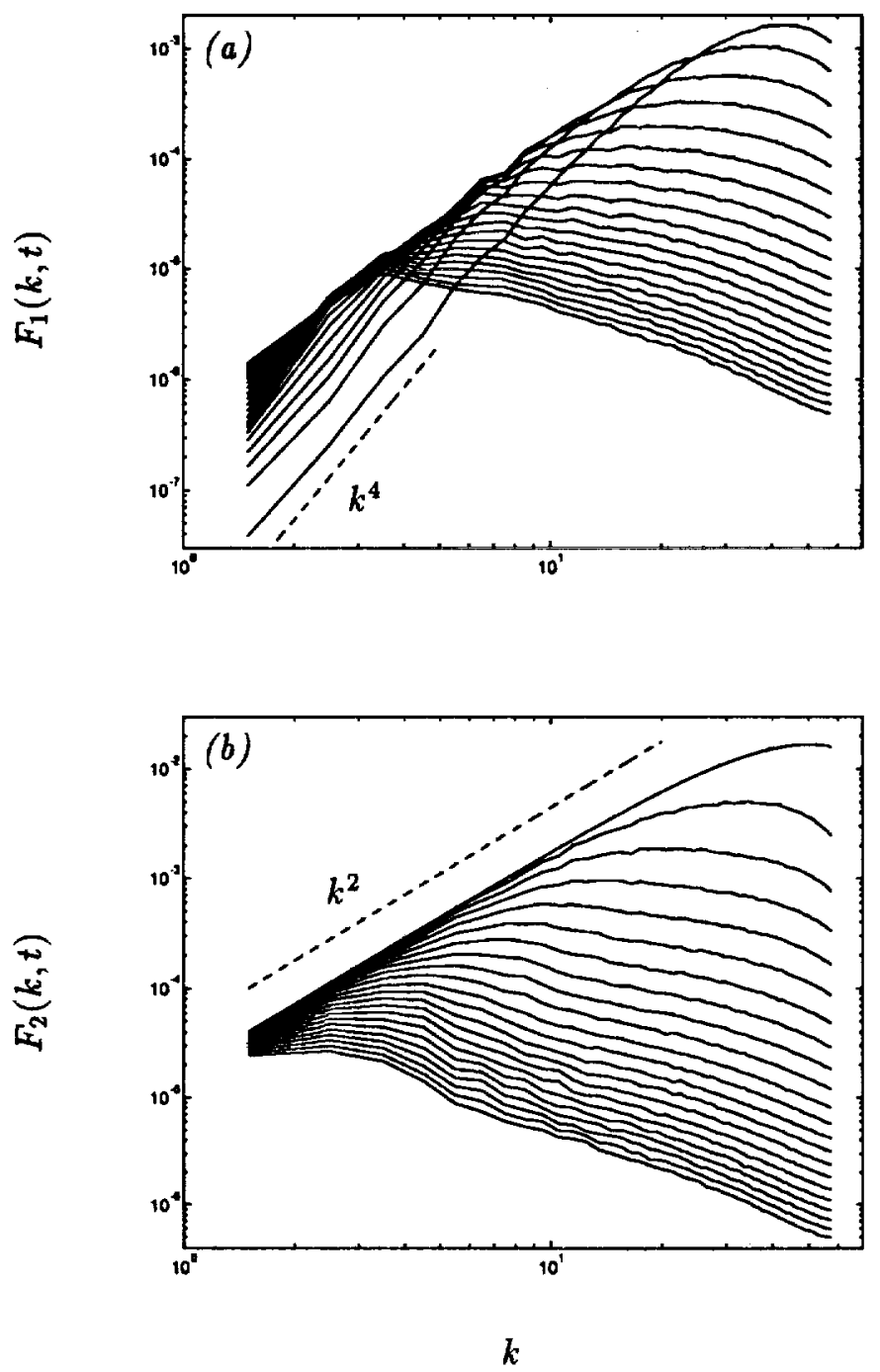

Figure 3. Time-evolution of the spectra defined in (22), for $s=2$ in (12) and impulsive forces parallel to the symmetry axis. (a) $F_{1}(k, t)$; (b) $F_{2}(k, t)$.

plot the $F_{1}(k, t)$ and $F_{2}(k, t)$ spectra computed from the flow simulation when the impulsive forces are parallel to the symmetry axis and $s=2$ in (12). At the initial instant, $F_{1}\left(k, 0^{+}\right)=0$, as seen from (20). The spectrum of $F_{1}(k, t)$ develops rapidly in time and a $k^{4}$ low wavenumber spectral form becomes apparent at small wavenumbers due to the nonlinear transfer of energy from small-to-large scales. The form of the $F_{2}(k, t)$ spectrum as $k \rightarrow 0$ is exactly $4 \pi B_{0} k^{2}$ for all times.

Using the invariant $B_{0}$, asymptotic similarity states can be constructed for the spectra $F_{1}(k, t)$ and $F_{2}(k, t)$ : 


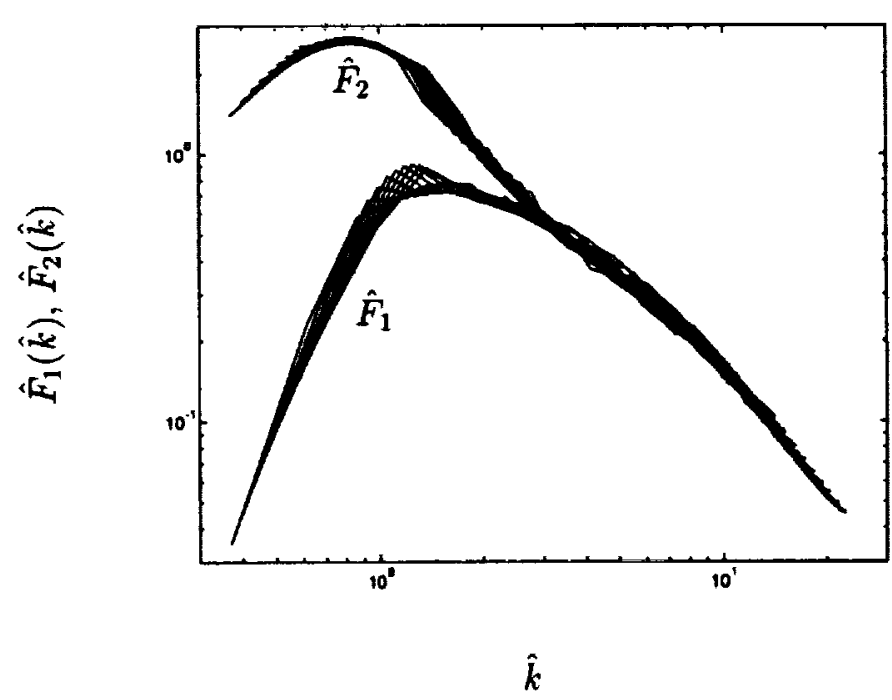

FIgURE 4. Time-evolution of the similarity spectra defined in (23), for $s=2$ in (12) and impulsive forces parallel to the symmetry axis.

$$
F_{1}(k, t)=B_{0}^{\frac{3}{6}} t^{-\frac{1}{6}} \hat{F}_{1}(\hat{k}), \quad F_{2}(k, t)=B_{0}^{\frac{3}{b}} t^{-\frac{1}{3}} \hat{F}_{2}(\hat{k}), \quad \hat{k}=B_{0}^{\frac{1}{5}} t^{\frac{2}{3}} k
$$

The last nine spectra of Figs. 3a and 3b are replotted on the same graph in Fig. 4 using the scalings given by $(23)$. An excellent collapse of the $\hat{F}_{1}(\hat{k})$ and $\hat{F}_{2}(\hat{k})$ spectra at different times is observed. Furthermore, the collapse of $\hat{F}_{1}(\hat{k})$ and $\hat{F}_{2}(\hat{k})$ together at large values of $\hat{k}$ indicates an approach of the small-scale turbulence to isotropy. Clearly, however, the flow remains anisotropic at the largest and energycontaining scales, as already indicated by the deviation of $\gamma$ from unity at large times. Apparently, the local value of $\gamma=8$ for the largest scales of the flow, and $\gamma=1$ for the smallest scales results in a balance in the energy containing scales of $\gamma \approx 1.5$.

An interesting result can be obtained from the similarity state given in (23) and confirmed by the results of Fig. 4 . The form of the $F_{1}(k, t)$ spectrum near $k=0$ can be written as

$$
F_{1}(k, t) \sim C(t) k^{4},
$$

where, as we have already noted, the $k^{4}$ spectrum arises from the nonlinear transfer from small-to-large scales. Substitution of this low wavenumber form of the $F_{1}(k, t)$ spectrum into (23), and using the independence of $\hat{F}_{1}(\hat{k})$ on $t$ and $B_{0}$, yields the exact result

$$
C(t) \propto B_{0}^{\frac{7}{8}} t^{\frac{4}{6}}
$$



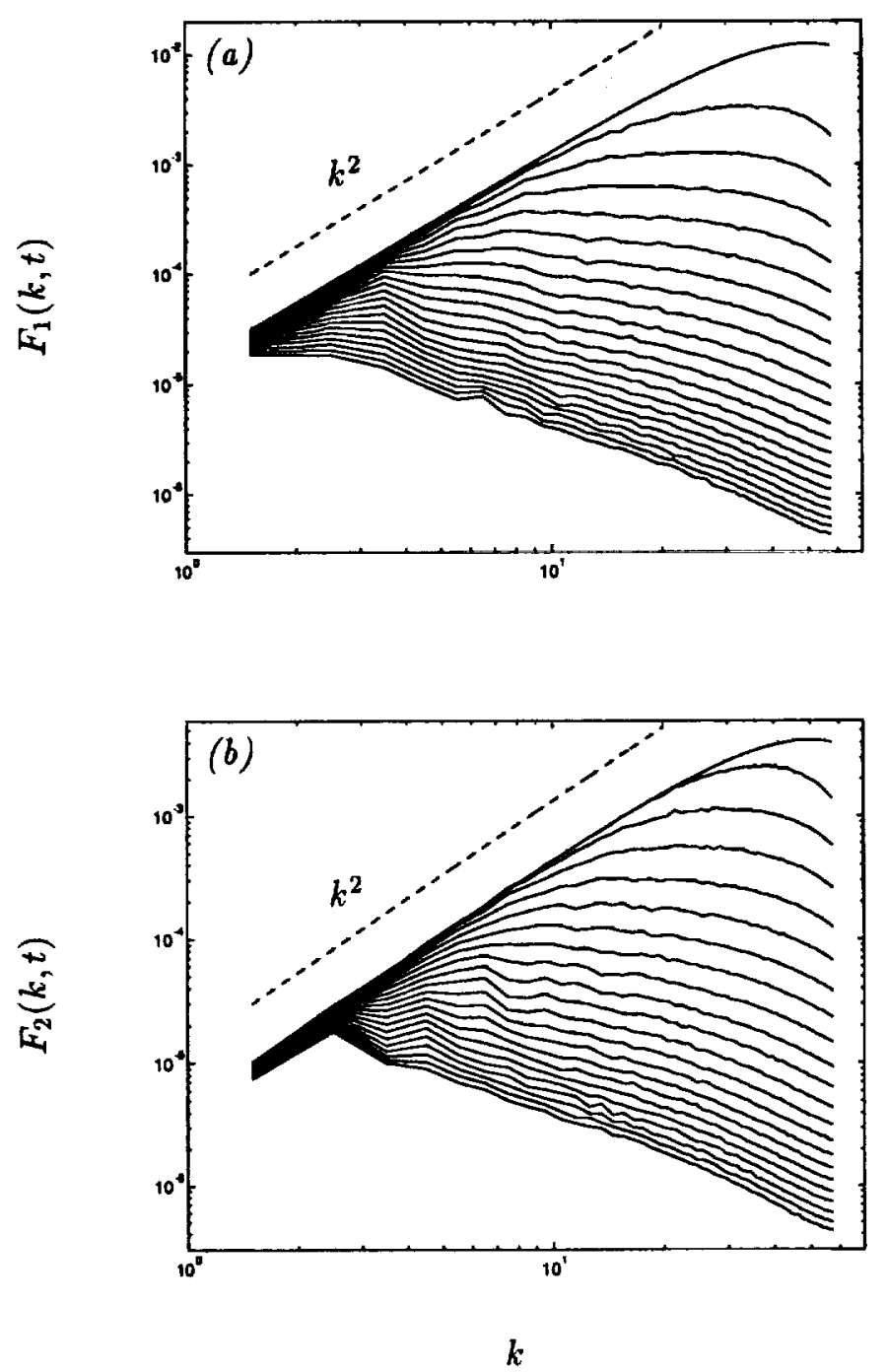

Figure 5. Time-evolution of the spectra defined in (22), for $s=2$ in (12) and impulsive forces perpendicular to the symmetry axis. (a) $F_{1}(k, t)$; (b) $F_{2}(k, t)$.

Hence, we have determined the large-scale structure of the turbulence characterized by the spectrum $F_{1}(k, t)$, even though this structure is a consequence of complicated non-linear interactions.

Similar ideas apply when the impulsive forces are perpendicular to the symmetry axis. In Figs. 5 , the time-evolution of the $F_{1}(k, t)$ and $F_{2}(k, t)$ spectra are plotted. The forms of the $F_{1}(k, t)$ and $F_{2}(k, t)$ spectra near $k=0$ are $F_{1}(k, t) \sim 3 \pi B_{0} k^{2}$ and $F_{2}(k, t) \sim \pi B_{0} k^{2}$ for all times. The rescaled spectra are plotted in Fig. 6 and again we find that the largest scales and the energy-containing scales of the flow remain anisotropic at the latest times, and the smallest scales approach isotropy. 


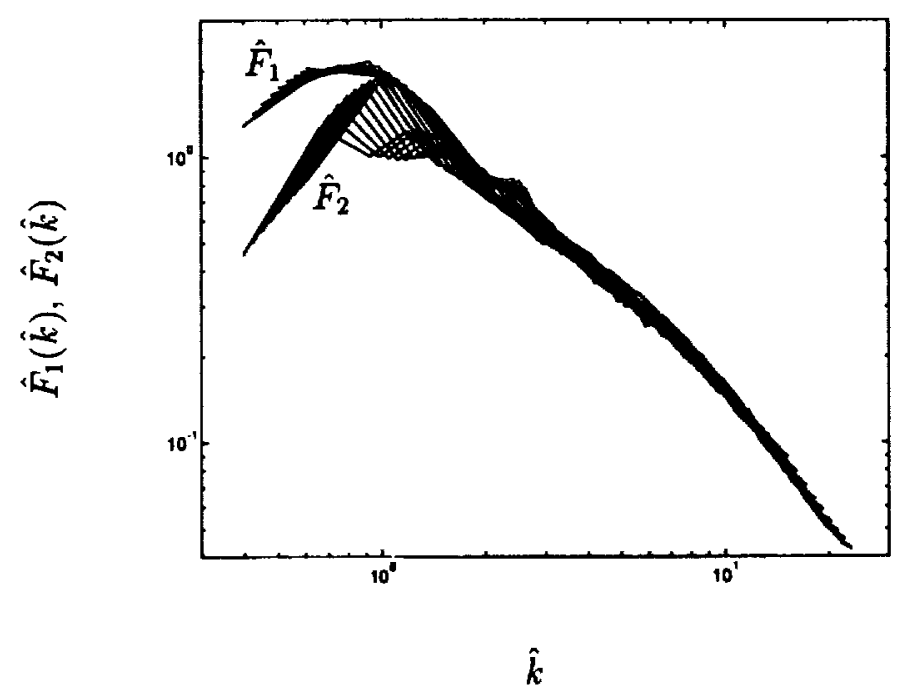

FIgURE 6. Time-evolution of the similarity spectra defined in (23), for $s=2$ in (12) and impulsive forces perpendicular to the symmetry axis.

When $s=4$ in (12) (and (14)), corresponding to a Batchelor-Proudman-type flow, the turbulence becomes isotropic asymptotically and the present results reduce to the decay of an isotropic turbulence, whose asymptotic similarity state has already been discussed in sufficient detail by Chasnov (1994).

\section{Conclusions}

We have thus demonstrated the existence of asymptotic similarity states of a decaying axisymmetric turbulence at high Reynolds numbers. A complete returnto-isotropy of the turbulence occurs in Batchelor-Proudman type flows but does not occur in Saffman-type flows due to the permanence of the anisotropic structure of the large scales. Simulations were performed based on initial flow fields which were generated by axisymmetric random impulsive forces acting at the initial instant. Such a turbulence generation mechanism has an analogy in active grid turbulence experiments, and it would be of interest to see if the results obtained here may be reproducible in the laboratory.

\section{Acknowledgments}

I wish to thank R. Rogallo and A. Wray for allowing me use of their homogeneous turbulence code for the Intel iPSC/860 hypercube machine at NAS. I would also like to thank C. Cambon, P. Durbin, N. Mansour, and K. Squires for useful discussions during the Summer Program. 


\section{REFERENCES}

Batchelor, G. K. And Proudman, I. 1956 The large-scale structure of homogeneous turbulence. Philos. Trans. R. Soc. London. 248, 369.

Chasnov, J. R. 1993 Computation of the Loitsianski integral in decaying isotropic turbulence. Phys. Fluids A. 5, 2579.

Chasnov, J. R. 1994 Similarity states of passive scalar transport in isotropic turbulence. Phys. Fluids. 6, 1036.

Chollet, J. P., AND Lesieur, M. 1981 Parameterization of small scales of three dimensional isotropic turbulence utilizing spectral closures. J. Atmos. Sci. 38, 2747.

Comte-Bellot, G. AND Corrsin, S. 1966 The use of a contraction to improve the isotropy of grid-generated turbulence. J. Fluid Mech. 25, 657.

Herring, J. R. 1974 Approach of axisymmetric turbulence to isotropy. Phys. Fluids. 17, 859.

Kolmogorov, A. N. 1941 On degeneration of isotropic turbulence in an incompressible viscous liquid. Dokl. Akad. Nauk. SSSR. 31, 538.

Kraichnan, R. H. 1976 Eddy viscosity in two and three dimensions. J. Atmos. Sci. 33, 1521.

Lesieur, M. 1990 Turbulence in Fluids. Kluwer Academic Publishers.

LOITSIANSKI, L. G. 1939 Some basic laws for isotropic turbulent flow. Trudy Tsentr. Aero.-Giedrodin. Inst. 440, 31.

Rogallo, R. S. 1981 Numerical experiments in homogeneous turbulence. NASA TM 81315.

SAFFMAN, P. G. 1967a The large-scale structure of homogeneous turbulence. $J$. Fluid Mech. 27, 581.

SaffMAN, P. G. 1967b Note on decay of homogeneous turbulence. Phys. Fluids. $10,1349$.

SchumanN, U. AND Herring, J. R. 1976 Axisymmetric homogeneous turbulence: a comparison of direct spectral simulations with the direct-interaction approximation. J. Fluid Mech. 76, 755.

Schumann, U. ANd Patterson, G. S. 1978 Numerical study of the return of axisymmetric turbulence to isotropy. J. Fluid Mech. 88, 711 . 
